\title{
Selenium-Bifidobacterium longum as a delivery system of endostatin for inhibition of pathogenic bacteria and selective regression of solid tumor
}

\author{
CHEN LI $^{1}$, XU CHEN ${ }^{1}$, LEI KOU ${ }^{1}$, BI HU ${ }^{1}$, LI-PING ZHU ${ }^{1}$, YAN-RONG FAN ${ }^{2}$, \\ ZHI-WEI WU ${ }^{3}$, JIAN-JUN WANG ${ }^{1}$ and GEN-XING XU ${ }^{3,4}$ \\ ${ }^{1}$ Department of Biological Science and Technology and State Key Laboratory of Pharmaceutical Biotechnology, \\ School of Life Sciences, Nanjing University, Nanjing 210093; ${ }^{2}$ School of Chemical Engineering, \\ Nanjing University of Science and Technology, Nanjing 210094; ${ }^{3}$ Center for Public Health Research, \\ Medical School, Nanjing University, Nanjing 210093; ${ }^{4}$ Jiangsu Research Center for \\ Gene Pharmaceutical Engineering and Technology, Suzhou 215128, P.R. China
}

Received July 21, 2009; Accepted September 8, 2009

DOI: 10.3892/etm_00000022

\begin{abstract}
Bifidobacterium longum (B. longum) as a delivery system for endostatin was shown to have definite antitumor effects. Moreover, it was found that the enrichment of selenium was able to enhance the immunity of mice. In order to further evaluate the safety and efficacy of $B$. longum carrying pBV22210-endostatin (B. longum-En) enriched with selenium (Se-B. longum-En), we determined the biochemical characteristics of Se-B. longum-En. We then investigated its effect on macrophage activity, as well as its inhibitory effect on the multiplication of pathogenic bacteria in vitro and the antitumor effects on murine hepatic (H22) tumor-bearing mice. The results showed that Se-B. longum-En exhibited similar biochemical characteristics to that of wild-type B. longum, i.e., Se- $B$. longum-En strongly enhanced macrophage phagocytosis in rats and inhibited the growth of pathogenic bacteria. In addition, Se-B. longum-En showed a definite inhibitory effect of tumor growth when $\mathrm{H} 22$ tumor-bearing mice were fed through oral or tail vein delivery. These results suggested that Se- $B$. longum is able to retain the advantages of wild-type $B$. longum and be used as a novel gene delivery system for liver cancer gene therapy.
\end{abstract}

Correspondence to: Professor Gen-Xing Xu, Center for Public Health Research Medical School, Nanjing University, 22 Hankou Road, Nanjing 210093, P.R. China

E-mail: genxingxu@nju.edu.cn

Professor Jian-Jun Wang, School of Life Sciences, Mailbox 426, Nanjing University, 22 Hankou Road, Nanjing 210093, P.R. China

E-mail: jjwang@nju.edu.cn

Key words: endostatin, Bifidobacterium longum, selenium, gene therapy

\section{Introduction}

Angiogenesis is required in the growth and metastasis of solid tumors. Extensive studies have been conducted on various angiogenic inhibitors. O'Reilly et al reported on such an angiogenic inhibitor, endostatin, which has a broad antitumor spectrum and low toxicity (1). Mammalian studies indicated that endostatin had few side effects and induced no drug resistance after prolonged use (2). These properties make endostatin a promising candidate as an antitumor agent.

A primary problem for cancer gene therapy is the lack of specificity in current delivery systems. Many solid tumors in rodents exhibit similar features of hypoxic regions as those in humans. Therefore, anaerobic non-pathogenic bacteria such as Bifidobacterium and Lactobacillus are regarded as favorable media for the delivery of specific tumor-inhibiting genes (3-6). Previous studies have shown that the role of Bifidobacterium longum (B. longum) as a delivery system for endostatin is tumor-specific with no toxicity $(7,8)$. Selenium (Se), essential micronutrient in the daily diet, was shown to beneficial to human health, especially in cancer chemoprevention. Some studies demonstrated that a Se supplement reduces carcinogenic risk $(9,10)$. Se is speculated to play a role by entering the protein as an amino acid selenocysteine (Sec), coded by UGA. Selenoproteins are associated with the deletion of carcinogen-initiated cells and the suppression of transformed cell expansion.

In the present study, Se was enriched to transformed $B$. longum carrying the shuttle vector pBV22210-endostatin (B. longum-En). Its biochemical characteristics and effect on pathogenic bacteria, as well as its antitumor effects on $\mathrm{Se}-B$. longum-En were determined on murine hepatic (H22) tumorbearing mice through oral and intravenous (i.v.) delivery. The results showed that Se- $B$. longum-En maintained its biochemical characteristics and exhibited inhibitory activity on tumor growth. 


\section{Materials and methods}

Plasmids, bacterial strains and reagents. A shuttle vector pBV22210 coding endostatin was constructed and transfected into B. longum-En (Xu et al of our laboratory) (8). Wildtype (WT) B. longum, shigella flexneri51571, salmonella typhimurium50115, escherichia coli44155 and pseudomonas aeruginosa03 were obtained from the Inner Mongolia Shuangqi Medical Industry Corporation (Inner Mongolia, P.R. China). Chicken blood was obtained from a vein under the wing from a $2 \mathrm{~kg}$ hen. Chicken red blood cells (RBC) were separated following centrifugation at $2000 \mathrm{~g}$ for $5 \mathrm{~min}$. Cyclophosphamide (CTX) was purchased from Shanghai Lianhua Pharmaceutical Co. Ltd. China (Shanghai, P.R. China). Ketamine was purchased from Shanghai First Biochemical Pharmaceutical Co. Ltd. China (Shanghai, P.R. China).

Animals and tumor cells. Male Kunming mice, aged 6-8 weeks ( $20 \pm 1 \mathrm{~g})$, were purchased from the animal center of Nanjing Medical University (Nanjing, P.R. China). Mice were fed with standard rodent diet and water ad libitum and kept in an animal facility, maintained at $21 \pm 2^{\circ} \mathrm{C}$ on a 12 -h light/dark cycle. H22 cells were supplied by the Shanghai Academy of Medical Industry (Shanghai, P.R. China). A liver tumor model was established by the subcutaneous injection of $\mathrm{H} 22$ tumor cells $\left(1 \times 10^{6} / 0.2 \mathrm{ml}\right)$ into the flank of each mouse.

Selenium enrichment to $B$. longum-En and selenium quantification. Selenium enrichment to B. longum-En was performed according to the previously established protocol (7). Briefly, sodium selenite was weighed and dissolved in $200 \mathrm{ml}$ TPY medium at a concentration of 5, 10, 15, 20 and $25 \mu \mathrm{g} / \mathrm{ml}$, respectively. Overnight cultivated B. longum-En in TPY medium was washed three times with phophatebuffered saline (PBS). The number of viable bacteria for Se- $B$. longum-En was measured according to the Manufacturing and Determining Regulations of Oral Live Bifidobacterium Preparatum in Regulations for Biological Products of People's Republic of China (11). The total selenium content in $B$. longum-En was determined according to the Determination of Selenium in foods (12). The inorganic and organic form of selenium content in B. longum-En was measured by atomic absorption spectrometry according to the appendix VIII D of Chinese Pharmacopeia (13).

Analysis of Se-B. longum-En. The identification of Se-B. longum-En was carried out by the Inner Mongolia Shuangqi Medical Industry Corporation (Inner Mongolia). Aerobic, dynamic, spore, catalase and gelatin liquefaction assays, as well as deoxidize by nitrate and carbohydrate fermentation were performed according to previously published protocols (14). B. longum-En and WT B. longum were used as controls.

Effect of measurements of Se-B. longum-En on macrophage phagocytotic activity. In order to determine the effect of Se- $B$. longum-En on immunity, we measured the macrophage phagocytotic activity in rats. Twenty-four Wistar rats (male, weighing $220 \pm 15 \mathrm{~g}$ ) were divided into three groups. Each group received dextrose-saline solution intraperitoneally (i.p.) on days 1-3, WT B. longum cells (i.p., days 1-3) and Se-B. longum-En cells (i.p., days 1-3), respectively. The rats in two of the treated groups were allergized using the method: $1 \mathrm{ml}$ liquid bacterium $\left[10^{8}\right.$ colony-forming unit (CFU)] dissolved in dextrose-saline solution was injected into each mouse on day 1, $2 \mathrm{ml}\left(10 \times 10^{8} \mathrm{CFU}\right)$ on day 2 , and $4 \mathrm{ml}\left(20 \times 10^{8} \mathrm{CFU}\right)$ on day 3. Rats in the dextrose-saline solution group were used as a negative control. On day 9, the peritoneal fluids of each group were collected for the preparation of macrophages. Briefly, $20 \mathrm{ml}$ of PBS (pH 7.0) were injected into rat abdominal cavities. The rats were anesthetized with ketamine (120 $\mathrm{mg} / \mathrm{kg})$. Ascites $(15 \mathrm{ml})$ were removed from each rat after 5 min cheirapsis. The ascites were washed three times with PBS before being adjusted to a concentration of $3 \times 10^{6}$ cell/ $\mathrm{ml}$. Macrophage suspension was then obtained. RBC suspension ( $1 \mathrm{ml}$; containing $2 \%$ chicken $\mathrm{RBC}$ ) was added to $1 \mathrm{ml}$ macrophage suspension and the mixture was centrifuged at $1000 \mathrm{~g}$ for $8 \mathrm{~min}$ after incubation at $37^{\circ} \mathrm{C}$ for $30 \mathrm{~min}$. The supernatant was discarded and precipitation was re-suspended before being plated onto the slides. The cells were stained and counted using the high microscope objective. The phagocytotic rate and index were calculated by the formula:

$$
\text { Rate of phagocytosis }=\frac{\text { no. of macrophage cells in } 200 \text { cells }}{200} \times 100 \%
$$

no. of chicken RBC

$$
\text { Phagocytosis index }=\frac{\text { phagocytized by } 200 \text { macrophage cells }}{200} \times 100 \%
$$

Co-cultivation of Se-B. longum-En and pathogenic bacteria. The shigella flexneri51571, salmonella typhimurium50115, escherichia coli44155 and pseudomonas aeruginosa03 were co-incubated with WT B. longum or Se-B. longum-En under aerobic conditions. The co-cultures were incubated overnight at $37^{\circ} \mathrm{C}$. Colonies on agar plates with or without $5 \mu \mathrm{g} / \mathrm{ml}$ chloramphenicol were counted at $0,12,24,48,72$ and $96 \mathrm{~h}$, respectively. Each pathogenic bacterium was cultured alone as a control under the same conditions.

Suppression of H22 tumor growth by Se-B. longum-En through oral delivery. The tumor-bearing mice were divided into five groups. Animals were treated with Se-B. longum-En [0.8 ml, intragastrically (i.g.), days 1-30], B. longum-En (0.8 $\mathrm{ml}$, i.g., days 1-30) and WT B. longum (0.8 ml, i.g., days 1-30), respectively. However, the positive control group was injected with CTX (30 mg/kg, i.p., days 1-7) and another group received $13 \%$ fat-free milk $(0.8 \mathrm{ml}$, i.g., days $1-30)$ as a negative control. Prior to injection, $B$. longums were washed three times with dextrose-saline solution and re-suspended in $13 \%$ fat-free milk to $7.5 \times 10^{8} \mathrm{CFU} / \mathrm{ml}$. Seventy-two hours after the last administration the animals were sacrificed and tumors were excised and weighed. The inhibition rate (IR) on the tumor growth was determined by the formula:

IR $=\frac{\text { tumor weight of control group }- \text { tumor weight of treatment group }}{\text { tumor weight of control group }} \times 100 \%$

Suppression of H22 tumor growth by Se-B. longum-En through tail vein delivery. The $\mathrm{H} 22$ tumor-bearing mice were randomly divided into five groups. Se- $B$. longum-En, $B$. 
A
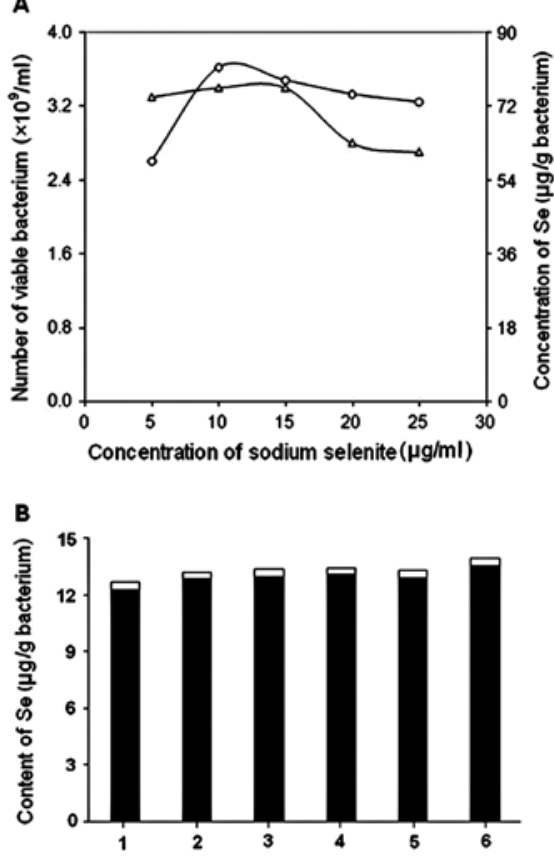

Figure 1. Selenium content in Se-B. longum-En. (A) The growth rates for Se- $B$. longum-En and selenium content changed by different concentrations of sodium selenite. Open triangles show the numbers of viable Se-B. longum-En and open circles show selenium content. (B) Selenium content in six samples of Se-B. longum-En. Six random samples (1-6) are shown. The white and black parts of the bars represent the inorganic and organic form of selenium in the samples, respectively.

longum-En, and WT B. longum were washed as mentioned above. These three groups were then re-suspended in dextrosesaline solution at a concentration of $2.5 \times 10^{8}$ cells $/ \mathrm{ml}$ before injection. Mice were injected with Se-B. longum-En (0.4 $\mathrm{ml}$, i.v., days 1-7), B. longum-En (0.4 ml, i.v., days 1-7), WT B. longum (0.4 ml, i.v., days 1-7), CTX (30 mg/kg, i.p., days 1-7) and dextrose-saline solution (0.4 ml/mice i.v., days 1-7), respectively. The animals were kept for an additional three days following the last injection, and were sacrificed seven days later. The weight of the excised tumors and IR of tumor growth were determined as described above.

Statistical analysis. The data were statistically analyzed using Student's t-test in both groups and the ANOVA test in multiple groups. Comparisons among the multiple groups were
A

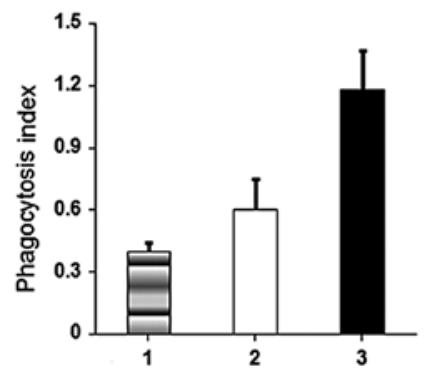

B

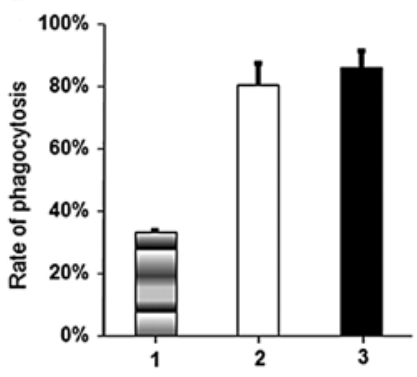

Figure 2. Enhancement of phagocytotic activity of macrophage by Se- $B$. longum-En. (A) Bars 1-3 show the rate of phagocytosis of rat macrophages in the negative control, WT B. longum and Se-B. longum-En groups, respectively. (B) Bars 1-3 show the phagocytotic indices of rat macrophages in the negative control, WT B. longum and Se-B. longum-En groups, respectively.

performed using the Student-Newman-Keuls Q-test. P $<0.05$ was considered to be significant.

\section{Results}

Selenium content in Se-B. longum-En. The growth curve and selenium content of Se-B. longum-En were measured. As shown in Fig. 1A, the growth curve of Se-B. longum-En and selenium content did not increase with the increasing concentration of sodium selenite. Instead, both showed their peak values when the concentration of sodium selenite reached $10 \mu \mathrm{g} / \mathrm{ml}$, suggesting that $10 \mu \mathrm{g} / \mathrm{ml}$ was the most suitable concentration to enrich Se by B. longum-En. Measurement of the selenium content in inorganic and organic forms was also performed. Fig. 1B shows that the percentage of organic selenium was $>96 \%$ of the total selenium content.

Biochemical characteristics of Se-B. longum-En. B. longum bacterium colonies were ivory white round scabrosities with a smooth surface. The diameter of these colonies ranged from 0.6 to $1.8 \mathrm{~mm}$. Se-B. longum-En exhibited the same biochemical characteristics as those of $B$. longum-En. In our study, both showed minor differences in carbohydrate fermentation to the WT B. longum. Se-B. longum-En and B. longum-En were not able to ferment arabinose and mannose, both of which were fermented in WT B. longum cells. These results

Table I. The main biochemical characteristics of (A) Se-B. longum-En, (B) B. longum-En and (C) WT B. longum.

\begin{tabular}{|c|c|c|c|c|c|c|c|c|c|c|c|c|c|c|c|c|c|c|}
\hline & \multirow[b]{2}{*}{1} & \multirow[b]{2}{*}{2} & \multirow[b]{2}{*}{3} & \multirow[b]{2}{*}{4} & \multirow[b]{2}{*}{5} & \multirow[b]{2}{*}{6} & \multirow[b]{2}{*}{7} & \multirow[b]{2}{*}{8} & \multicolumn{10}{|c|}{ Carbohydrate fermentation } \\
\hline & & & & & & & & & 9 & 10 & 11 & 12 & 13 & 14 & 15 & 16 & 17 & 18 \\
\hline A & $\mathrm{G}^{+}$ & - & + & - & - & - & - & - & + & - & + & - & - & - & - & + & - & - \\
\hline B & $\mathrm{G}^{+}$ & - & + & - & - & - & - & - & + & - & + & - & - & - & - & + & - & - \\
\hline $\mathrm{C}$ & $\mathrm{G}^{+}$ & - & + & - & - & - & - & - & + & + & + & - & - & - & + & + & - & - \\
\hline
\end{tabular}

1, Gram staining; 2, aerobic assay; 3, anaerobic assay; 4, spore assay; 5, dynamic assay; 6, catalase assay; 7, gelatin liquefaction assay; 8, deoxidize by nitrate; 9 , glucose; 10, arabinose; 11, lactose; 12 , cellobiose; 13 , starch; 14, esculin; 15 , mannose; 16, melibiose; 17, trehalose and 18, melezitose. (-), Negative reaction; (+), positive reaction. 
A1

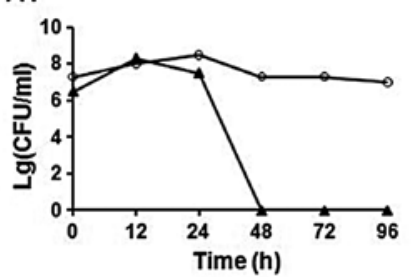

A2

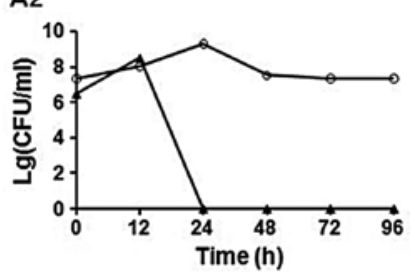

A3

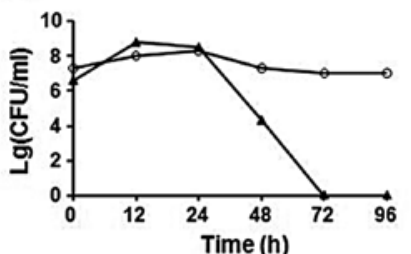

A4

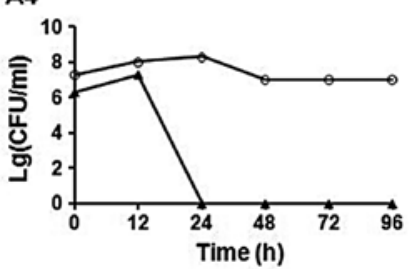

B1

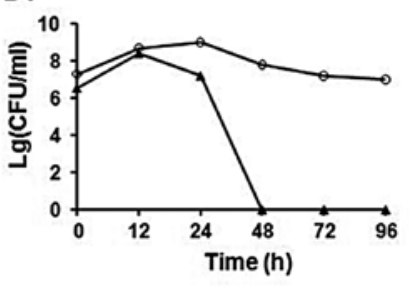

B2

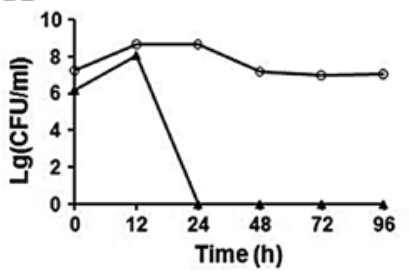

B3

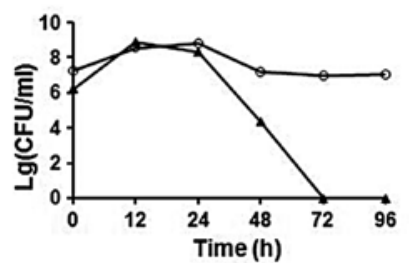

B4

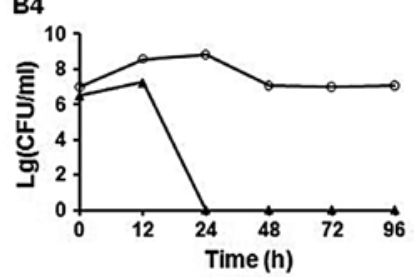

Figure 3. Growth curves of four pathogenic strains co-cultured with WT $B$. longum and Se-B. longum-En, respectively, are shown. A1-4 show the growth curve of shigella flexneri51571, salmonella typhimurium50115, escherichia coli44155 and pseudomonas aeruginosa03 cultured alone (open circle) or co-cultured with WT B. longum (filled triangle), respectively. B1-4 show the growth curves of shigella flexneri51571, salmonella typhimurium50115, escherichia coli44155 and pseudomonas aeruginosa03 cultured alone (open circle) or co-cultured with Se-B. longum-En (filled triangle), respectively.

suggested that gene transfection and selenium enrichment had little influence on the natural characteristics of B. longum. The results are shown in Table I.

Enhancement in phagocytotic activity of macrophage by Se-B. longum-En. Fig. 2 shows that the rates of phagocytosis in the transformed B. longum (Se-B. longum-En and B. longum-En) group were 46.72 and $52.40 \%$, respectively. These rates were higher than those in WT B. longum. Selenium enrichment to $B$. longum-En markedly enhanced the phagocytotic activity of rat macrophages compared with $B$. longum-En and WT $B$. longum, as is shown by the phagocytotic indices of $1.18,0.60$ and 0.40 , respectively.

Inhibition of pathogenic bacteria by Se-B. longum-En in vitro. Four pathogenic bacteria strains were strongly inhibited by co-cultivation with either WT B. longum or Se-B. longum-En (Fig. 3). Salmonella typhimurium50115 and pseudomonas aeruginosa03 were not detected $24 \mathrm{~h}$ after co-cultivation with WT B. longum or Se-B. longum-En. No colony of shigella
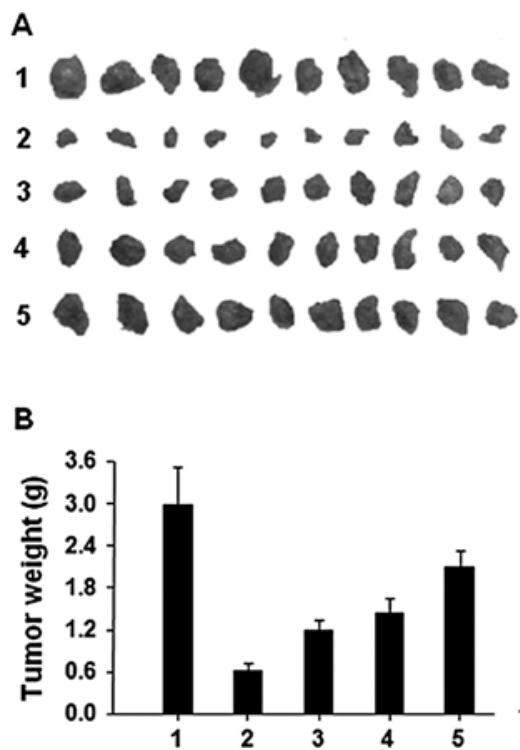

Figure 4. Inhibition of H22 tumor growth with Se-B. longum-En by intra-gastric administration. (A) Rows 1-5 show tumors excised from mice treated with $13 \%$ fat-free milk, CTX, B. longum-En, Se-B. longum-En and WT B. longum, respectively. (B) The average tumor weight of each group is shown. Bars 1-5 are the average weight of tumors in Rows 1-5.
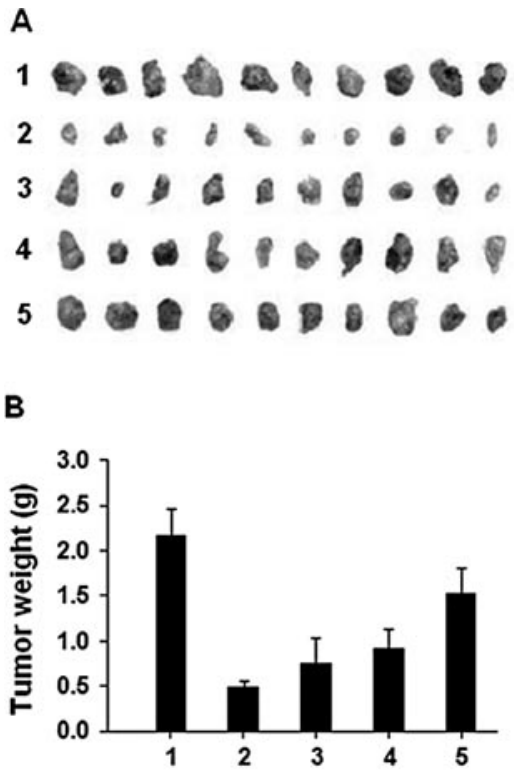

Figure 5. Inhibition of $\mathrm{H} 22$ tumor growth with Se-B. longum-En by i.v. administration. (A) Rows 1-5 show tumors excised from mice treated with dextrose-saline solution, CTX, B. longum-En, Se-B. longum-En and WT $B$. longum, respectively. (B) The average tumor weight of each group is shown. Bars 1-5 show the average weight of tumors in Rows 1-5.

flexneri51571 was found $48 \mathrm{~h}$ after co-cultivation, nor was any colony of escherichia coli44155 observed $72 \mathrm{~h}$ after co-cultivation. No significant difference was noted between the WT B. longum and Se-B. longum-En groups.

Suppression of H22 tumor growth with Se-B. longum-En by intra-gastric administration. Tumors excised from the mice are shown in Fig. 4A. Fig. 4B shows that mice treated with transformed B. longum grew more slowly than those treated with $13 \%$ fat-free milk. Compared with the negative control 
group, tumor weights were significantly reduced by $51.8 \%$ $(\mathrm{P}<0.00001)$ in the B. longum-En and by $60.1 \%(\mathrm{P}<0.00001)$ in Se- $B$. longum-En groups, respectively. Selenium enrichment to $B$. longum-En resulted in $\sim 10 \%(\mathrm{P}=0.0087)$ enhancement of the antitumor effect of B. longum-En. Fat-free milk showed partial tumor suppression with an IR of $29.7 \%(\mathrm{P}=0.00057)$.

Suppression of H22 tumor growth by Se-B. longum-En through i.v. administration. The inhibition of $\mathrm{H} 22$ tumor growth was determined by treating the $\mathrm{H} 22$ tumor mice with Se- $B$. longum-En. Tumors excised from the mice and tumor weights of each group are shown in Fig. 5. Tumor growth, measured in weight, was inhibited by $65.16(\mathrm{P}<0.00001)$, $57.21(\mathrm{P}<0.00001)$ and $29.85 \%(\mathrm{P}=0.00013)$ in the $\mathrm{Se}-B$ longum-En, B. longum-En and WT B. longum groups, respectively, as compared with the dextrose-saline solution group. The positive control group inhibited tumor growth by $77.59 \%(\mathrm{P}<0.00001)$. The results suggested that transformed B. longum cells significantly inhibited growth of the H22 tumor. No statistical difference was noted between the $B$. longum-En and Se-B. longum-En groups $(\mathrm{P}=0.143)$ though the tumor IR was $8 \%$ higher in the $\mathrm{Se}-B$. longum-En group.

\section{Discussion}

As a non-toxic angiogenic inhibitor with no known side effects or drug resistance, endostatin was considered to be a novel candidate for cancer therapy (15). It can activate the downstream Wnt/-catenin pathway and induce reorganization in endothelial cells by binding to several receptors on endothelial cells including $\alpha 5 \beta 1, \alpha 5 \beta 3$ and $\alpha 5 \beta 5$ integrins, heparin sulfate, tropomyosin, caveolin-1, vascular endothelial growth factor receptor-1 (VEGFR-1; Flt-1) and VEGFR-2 (Flk-1). The Wnt/catenin pathway and reorganization in endothelial cells were considered to play a role in inhibiting the proliferation and metastasis of endothelial cells, thereby inducing tumor apoptosis (16). Several studies have demonstrated that endostatin has an inhibitory effect on vessel generation in tumors in a dose-dependent manner without apparent side effects or drug resistance (17-19). Endostatin efficacy on many tumor cells, such as renal carcinoma, bladder cancer, Heps liver cancer, C6 Glioma, K1735 melanoma and C666-1 nasopharyngeal carcinoma has been noted in gene therapy studies (14,20-25). However, endostatin failed to show a similar effect on acute lymphocytic leukemia and neuroblastoma (26-31).

The greatest obstacle of gene therapy is the lack of an efficient delivery system. As probiotics, bifidobacteria have been widely used in the prevention and treatment of gastrointestinal disorders. In previous years, the anaerobic property of bifidobacteria was explored for cancer gene therapy (32-36). Yazawa et al observed that B. longum was not detected in normal tissues but selectively germinated in tumors after i.v. administration. Further studies showed that B. longum was able to be used as a specific gene therapy delivery system for breast tumors $(5,36)$. Fu et al used transformed B. longum carrying shuttle vector pBV220 $\left(\mathrm{Amp}^{+}\right)$encoding human endostatin as an oral delivery system. The results of $\mathrm{Fu}$ et al showed that B. longum-pBV220-En strongly inhibited the growth of solid liver tumor in a HepG2 liver tumor xenograft model and prolonged survival in experimental animals (7). Xu et al developed a more stable B. longum-pBV22210-En constructor that showed a stronger suppression of tumor growth in xenograft models (8).

Selenium was shown to benefit patients subjected to cancer chemoprevention (37). Furthermore, selenium supplementation enhanced lymphocyte activities and reduced the incidence of lung cancer (38-40). Studies have demonstrated that selenocysteine is responsible for the majority of $\mathrm{Se}$ biological effects (41). Presumably, selenoproteins in the form of selenocysteine or the S-Se-S compound were involved in the reversible cysteine/disulfide transformation which occurred in a number of redox-regulated proteins. The monomethylated form of Se was associated with the apoptosis-inducing process in transformed cells (10). In our previous study, Fu et al observed that the antitumor effect was enhanced when B. longum-pBV220-En was enriched with Se. In addition, selenium enrichment to $B$. longum-pBV220-En improved natural killing cell activity, stimulated T-cell proliferation and promoted the activity of IL- 2 and TNF- $\alpha$ in BALB/c mice (7). It has been known that Se can only be utilized in its organic form in vivo. We determined the most suitable concentration of sodium selenite for enriched and quantified selenium in its organic form. We also observed that Se-B. longum-En markedly enhanced the phagocytotic activity of rat macrophages with a phagocytotic index of 1.18 , which was significantly higher than that of B. longum-En (0.60) and WT B. longum (0.40).

Maintaining the inherent characteristics of B. longum is important for retaining the physiological role of B. longum, which contributes to the advantages of $B$. longum as a gene delivery system, including the enhancement of immunity and selective location in tumors. Carbohydrate fermentation is considered to be of great significance in identifying the bacterium strain. The results showed that B. longum-En and Se-B. longum-En differed from WT B. longum in carbohydrate fermentation in arabinose and mannose (Table I). This difference indicated that B. longum-En and Se-B. longum-En adopted similar morphological and biochemical characteristics as those of WT B. longum. It was reported that the fermentation of L-arabinose, gluconate, inulin, lactose, D-mannose, methyl a-D-glucoside, ribose, salicin, trehalose or xylose was variable in B. longum (42). Results of additional indices, including the aerobic, dynamic and spore assays, showed that there was no difference between B. longum-En, Se-B. longum-En and WT $B$. longum. In addition, we examined the in vitro activity of transformed $B$. longum in the inhibition of pathogenic bacteria and the results showed that both $B$. longum-En and Se- $B$. longum-En markedly enhance phagocytotic activity when compared with WT B. longum. However, no difference to WT B. longum was noted in the inhibition of pathogenic bacterium, suggesting that selenium enrichment was not able to enhance the regressive effect on the pathogenic bacteria of $B$. longum-En in vitro. Our results indicated that the gene transfection of endostatin and selenium enrichment had little influence on the characteristics of $B$. longum. Thus, it is important to further evaluate the safety and availability of Se-B. longum-En.

The present study showed that Se-B. longum-En resulted in an approximately $10 \%$ higher tumor IR than that of $B$. longum-En on tumor-bearing mice through intra-gastric $(\mathrm{P}=0.0087)$ and i.v. $(\mathrm{P}=0.143)$ administration. The higher 
IR suggested that the enhancement of immunity and downregulation of related gene expression by the transfection of endostatin and selenium enrichment is a possible mechanism of improvement on tumor inhibition.

In conclusion, selenium enrichment to B. longum-En not only retained the biochemical properties of $B$. longum but also enhanced the $B$. longum immune stimulatory activities, and increased the antitumor effect of $B$. longum-En. These results suggested that Se-B. longum be used as an effective delivery system of endostatin for the inhibition of liver tumors.

\section{Acknowledgements}

We thank Chengyun Deng, Qian Feng (Inner Mongolia Shuangqi Pharmaceutical Co. Ltd., P.R. China) and Yefen Xu who constructed the vector B. longum-pBV22210-endostatin. This work was supported by grant 2006AA02Z19E of the 863 Project from the State Ministry of Science and Technology of China, the 985-II Project from Nanjing University and grant BK2008150 from the Natural Science Foundation of Jiangsu Province to G.X.X. This work was also supported by grants 30670671 from the National Natural Science Foundation of China and BK2006713 from the Natural Science Foundation of Jiangsu Province, P.R. China to J.J.W.

\section{References}

1. O'Reilly MS, Boehm T, Shing Y, et al: Endostatin: an endogenous inhibitor of angiogenesis and tumor growth. Cell 88: 277-285, 1997.

2. O'Reilly MS, Holmgren L, Chen C and Folkman J: Angiostatin induces and sustains dormancy of human primary tumors in mice. Nat Med 2: 689-692, 1996.

3. Dachs GU, Patterson AV, Firth JD, Ratcliffe PJ, Townsend KM, Stratford IJ and Harris AL: Targeting gene expression to hypoxic tumor cells. Nat Med 3: 515-520, 1997.

4. Kimura NT, Taniguchi S, Aoki K and Baba T: Selective localization and growth of Bifidobacterium bifidum in mouse tumors following intravenous administration. Cancer Res 40: 2061-2068, 1980.

5. Yazawa K, Fujimori M, Amano J, Kano Y and Taniguchi S: Bifidobacterium longum as a delivery system for cancer gene therapy: selective localization and growth in hypoxic tumors. Cancer Gene Ther 7: 269-274, 2000.

6. Argnani A, Leer RJ, van Luijk N and Pouwels PH: A convenient and reproducible method to genetically transform bacteria of the genus Bifidobacterium. Microbiology 142: 109-114, 1996.

7. Fu GF, Li X, Hou YY, Fan YR, Liu WH and Xu GX: Bifidobacterium longum as an oral delivery system of endostatin for gene therapy on solid liver cancer. Cancer Gene Ther 12: 133-140, 2005.

8. Xu YF, Zhu LP, Hu B, Fu GF, Zhang HY, Wang JJ and Xu GX: A new expressing plasmid in Bifidobacterium Longum as a delivery system of endostatin for cancer gene therapy. Cancer Gene Ther 14: 151-157, 2007.

9. Hatfield DL and Gladyshev VN: How selenium has altered our understanding of the genetic code. Mol Cell Biol 22: 3565-3576, 2002.

10. Ganther HE: Selenium metabolism, selenoproteins and mechanisms of cancer prevention: complexities with thioredoxin reductase. Carcinogenesis 20: 1657-1666, 1999.

11. The Standardization Committee for Biological Products of People's Republic of China: Manufacturing and determining regulations of oral live bifidobacterium preparatum. In: Regulations for Biological Products of People's Republic of China. Chemical Industry Press, Beijing, pp424-429, 2000.

12. The State Standardization Management Committee of the People's Republic of China: Determination of selenium in foods. In: Modern Health Inspection. People's Health Press, Beijing, pp665-666, 2005.
13. The State Pharmacopoeia Committee of the People's Republic of China: Appendix VIII D: determination of selenium. In: Pharmacopoeia of the People's Republic of China. 2005 edition, Part II. Chemical Industry Press, Beijing, pp269, 2005.

14. Li X, Fu GF, Fan YR, Liu WH, Liu XJ, Wang JJ and Xu GX: Bifidobacterium adolescentis as a delivery system of endostatin for cancer gene therapy: selective inhibitor of angiogenesis and hypoxic tumor growth. Cancer Gene Ther 10: 105-111, 2003.

15. Yamaguchi N, Anand-Apte B, Lee M, et al: Endostatin inhibits VEGF-induced endothelial cell migration and tumor growth independently of zinc binding. EMBO J 18: 4414-4423, 1999.

16. Folkman J: Antiangiogenesis in cancer therapy - endostatin and its mechanisms of action. Exp Cell Res 312: 594-607, 2006.

17. Bloch W, Huggel K, Sasaki T, et al: The angiogenesis inhibitor endostatin impairs blood vessel maturation during wound healing. FASEB J 14: 2373-2376, 2000.

18. Boehm T, Folkman J, Browder T and O'Reilly MS: Antiangiogenic therapy of experimental cancer does not induce acquired drug resistance. Nature 390: 404-407, 1997.

19. Yin G, Liu W, An P, et al: Endostatin gene transfer inhibits joint angiogenesis and pannus formation in inflammatory arthritis. Mol Ther 5: 547-554, 2002.

20. Blezinger P, Yin G, Xie L, Wang J, Master M, Bishop JS and Min W: Intravenous delivery of an endostatin gene complexed in cationic lipid inhibits systemic angiogenesis and tumor growth in murine models. Angiogenesis 3: 205-210, 1999.

21. Sauter BV, Martinet O, Zhang WJ, Mandeli J and Woo SLC: Adenovirus-mediated gene transfer of endostatin in vivo results in high level of transgene expression and inhibition of tumor growth and metastases. Proc Natl Acad Sci USA 97: 4802-4807, 2000.

22. Kikuchi E, Menendez S, Ohori M, Cordon-Cardo C, Kasahara N and Bochner BH: Inhibition of orthotopic human bladder tumor growth by lentiviral gene transfer of endostatin. Clin Cancer Res 10: $1835-1842,2004$.

23. Read TA, Farhadi M, Bjerkvig R, Olsen BR, Rokstad AM, Huszthy PC and Vajkoczy P: Intravital microscopy reveals novel antivascular and antitumor effects of endostatin delivered locally by alginate-encapsulated cells. Cancer Res 61: 6830-6837, 2001.

24. Kiriseh M, Weigel P, Pinzer T, Carroll RS, Black PM, Schackert H and Schackert G: Therapy of hematogenous melanoma brain metastases with endostatin. Clin Cancer Res 11: 1259-1267, 2005.

25. Li XP, Li CYS, Li XH, et al: Inhibition of human nasopharyngeal carcinoma growth and metastasis in mice by adenovirus-associated virus-mediated expression of human endostatin. Mol Cancer Ther 5: 1290-1298, 2006.

26. Pawliuk R, Bachelot T, Zurkiya O, Eriksson A, Cao Y and Leboulch P: Continuous intravascular secretion of endostatin in mice from transduced hematopoietic stem cells. Mol Ther 5: 345-351, 2002.

27. Cui R, Takahashi K, Takahashi F, Tanabe KK and Fukuchi Y: Endostatin gene transfer in murine lung carcinoma cells induces vascular endothelial growth factor secretion resulting in up-regulation of in vivo tumorigenecity. Cancer Lett 232: 262-271, 2006.

28. Kuo CJ, Farnebo F, Yu EY, et al: Comparative evaluation of the antitumor activity of antiangiogenic proteins delivered by gene transfer. Proc Natl Acad Sci USA 98: 4605-4610, 2001.

29. Eisterer W, Jiang X, Bachelot T, et al: Unfulfilled promise of endostatin in a gene therapy-xenotransplant model of human acute lymphocytic leukemia. Mol Ther 5: 352-359, 2002.

30. Jouanneau E, Alberti L, Nejjari M, et al: Lack of antitumor activity of recombinant endostatin in a human neuroblastoma xenograft model. J Neurooncol 51: 11-18, 2001.

31. Marshall E: Cancer therapy: setbacks for endostatin. Science 295: 2198-2199, 2002.

32. Rossi M, Brigidi P and Matteuzzi D: Improved cloning vectors for Bifidobacterium spp. Lett Appl Microbiol 26: 101-104, 1998.

33. Rossi M, Brigidi P, Rodriguez AGV and Matteuzzi D: Characterization of the plasmid pMB1 from Bifidobacterium longum and its use for shuttle vector construction. Res Microbiol 147: 133-143, 1996.

34. Nakamura T, Sasaki T, Fujimori M, et al: Cloned cytosine deaminase gene expression of Bifidobacterium longum and application to enzyme/pro-drug therapy of hypoxic solid tumors. Biosci Biotechnol Biochem 66: 2362-2366, 2002.

35. Yi C, Huang Y, Guo ZY and Wang SR: Antitumor effect of cytosine deaminase/5-fluorocytosine suicide gene therapy system mediated by Bifidobacterium infantis on melanoma. Acta Pharmacol Sin 26: 629-634, 2005. 
36. Yazawa K, Fujimori M, Nakamura T, et al: Bifidobacterium longum as a delivery system for gene therapy of chemically induced rat mammary tumors. Breast Cancer Res Treat 66 : 165-170, 2001

37. Shamberger RJ and Frost DV: Possible protective effect of selenium against human cancer. Can Med Assoc J 104: 682, 1969.

38. Cheng DS, Deng YP and Lu XY: Effect of selenium supplementation on transplantive tumor growth and lymphocyte functions in mice. Acta Univ Sci Med Chongqing 25: 41-43, 2000.

39. Zhao R, Yu BM, Zhang GC, et al: Selenium enhancing the inhibitory effect of lymphocytes on the growth of colorectal neoplasm in nude mice. Chin J Exp Surg 17: 302-303, 2000.
40. Reid ME, Duffield-Lillico AJ, Garland L, Turnbull BW, Clark LC and Marshall JR: Selenium supplementation and lung cancer incidence: an update of the nutritional prevention of cancer trial. Cancer Epidemiol Biomarkers Prev 11: 1285-1291, 2002.

41. Hatfield DL and Gladyshev VN: How selenium has altered our understanding of the genetic code. Mol Cell Biol 22: 3565-3576, 2002.

42. Zhu L, Li W and Dong X: Species identification of genus Bifidobacterium based on partial HSP60 gene sequences and proposal of Bifidobacterium thermacidophilum subsp. porcinum subsp. nov. Int J Syst Evol Microbiol 53: 1619-1623, 2003. 